\section{Dr. Chogle replies}

To the Editor:

Neuromyelitis optica (NMO) is a demyelinating, organ-specific, autoimmune disorder that preferentially targets the optic nerve and spinal cord. Since the discovery and validation of NMO-IgG serum antibodies in patients with $\mathrm{NMO}$, the disorder has been considered a separate disease entity from multiple sclerosis (MS). NMO-IgG antibodies target aquaporin-4 (AQP4), the predominant water-channel protein within the central nervous system. AQP4 antibodies have also been described in formes frustes of NMO, longitudinally extensive transverse myelitis (LETM), and recurrent optic neuritis $(\mathrm{ON})^{1}$. The specificity of NMO-IgG antibodies for the disease led to the addition of NMO-IgG antibodies to the diagnostic criteria of $\mathrm{NMO}^{2}$. Incomplete forms of $\mathrm{NMO}$ have also been recognized, and in such patients the presence of NMO-IgG antibody positivity increases the risk of progression and has resulted in the designation of NMO spectrum disorders (NMOSD). The diagnostic criteria for NMOSD have also been recently defined ${ }^{3}$. Several case series have been described regarding the coexistence of connective tissue disease (CTD), and this has spurred interest in NMO/NMOSD in the field of rheumatology ${ }^{3}$. The 2 most commonly reported coexistent CTD are Sjögren's syndrome (SS) and systemic lupus erythematosus (SLE).

Sciascia, et $a l^{4}$ now describe a case of rhupus syndrome (RS) with high-titer AQP4 antibodies and associated myelitis. This case is an important addition to the literature on overlap CTD and NMO/NMOSD and highlights the importance of formulating accurate diagnostic and therapeutic plans in such overlap syndrome cases.

An editorial in The Journal points out that there is evidence supporting the existence of RS as a true overlap syndrome, with at least 3 biological markers that are hallmarks of rheumatoid arthritis (RA) and are present in RS but not in SLE 5 . These markers are (1) anticitrullinated protein antibodies, (2) adequate C-reactive protein response, and (3) genetic risk profile of RA. We used these 3 biological markers to diagnose RS in our patient who developed lupus erythematosus tumidus several years after anti-tumor necrosis factor therapy for RA. Incidentally, our patient had clinical, serological features of SS with anti-Ro antibodies with HLA class II genotype DQB $1 * 02$ and DRB $1 * 03$, a well established association of anti-Ro antibodies ${ }^{6}$.

Our recent patient with $\mathrm{RS}^{6}$ had no systemic features, while the patient with RS reported by Sciascia, et $a l^{4}$ had features of neuromyelitis with high-titer AQP4 antibodies. It would be interesting to know whether this patient's serum showed presence of anti-Ro antibodies, and the HLA class II genotype of this patient. Clinical signs of NMO or NMO-like disease have been reported with or following symptoms of SS. SS is an autoimmune disease characterized by inadequate tear and saliva production and a lymphocyte infiltration of exocrine glands, especially the lacrimal and salivary glands, which express AQP5 on the apical membrane and AQP4 on lateral membranes. Patients with SS typically have autoantibodies that bind the nuclear antigens and RNA-binding proteins Ro52, Ro60, and LA487. In the recently reported case series on NMOSD patients with CTD and myelitis, the presence of ON, NMO-IgG, and full-spectrum NMO occurred only in patients with $\mathrm{SS}^{3}$. These findings raise the question of whether an association exists between NMO and CNS disease seen in SS. The authors of this case series have commented that definitive evidence for an association between NMO and SS has not yet been established, and mechanistic studies are needed to explain the potential connection between the 2 diseases $^{3}$.

The genetic link between AQP4-specific T cells and NMO has also been studied. A hypothesis has been put forward that AQP4-specific T cells are present in patients with NMO-IgG because AQP4 is a protein antigen that requires T cell help for induction of AQP4-specific B cells. An MHC class II linkage (HLA, DPA1*0202, DPB1 0501, reactive with myelin basic protein) has been identified in patients (90\%) with Asian MS that some consider $\mathrm{NMO}^{7}$.

As reported by Sciascia, $e t a l^{4}$, several tools are useful for accurate diagnosis of NMOSD in a case of RS. These are contrast magnetic resonance imaging (MRI) of the brain and suspected area of cord involvement, lumbar puncture, visually evoked potentials in cases of suspected ON, and serum NMO-IgG testing. Brain imaging is an important tool in distinguishing MS from NMO/NMOSD; a brain MRI that is consistent with MS provides strong evidence against the diagnosis of NMO/NMOSD. Spinal MRI is invaluable in determining the presence of myelitis and allowing for classification into transverse myelitis and longitudinal myelitis (LM). LM is a characteristic finding in NMO/NMOSD and its presence may be used to differentiate from myelitis in classic MS, which rarely extends beyond a single vertebral segment ${ }^{3}$.

There are no studies directly evaluating optimal therapy in acute myelitis or ON secondary to $\mathrm{NMO}^{3}$. Some experts have advocated early use of B cell-depleting therapies as the standard care in patients with $\mathrm{NMO}^{8}$. In addition, the use of cyclophosphamide as an induction agent has been described in case reports of NMO based on its ability to deplete B cells and its effectiveness in SLE-related and SS-related myelitis ${ }^{3}$. Maintenance therapy with immunosuppressive medications is recommended for relapse prevention. Prednisone, azathioprine, and mycophenolate mofetil have all been effective in disease stabilization and relapse reduction in uncontrolled trials of $\mathrm{NMO}^{9}$.

That NMO/NMOSD can exist in isolation or in conjunction with systemic CTD such as SS or SLE has been documented. The case report by Sciascia, et al adds RS to the list of coexistent CTD, and highlights that rheumatologists should have a prominent role in the diagnostic evaluation of NMO/NMOSD to accurately identify or exclude CTD such as SS, SLE, or RS.

ARUN R. CHOGLE, MD, Division of Rheumatology, Sir Hurkisondas Nurrotumdas Hospital and Research Centre, Raja Rammohan Roy Road, Mumbai 400 004, India. Address correspondence to Dr. Chogle; E-mail: archogle@vsnl.net/arunchogle@yahoo.com

\section{REFERENCES}

1. Jarius S, Jacobi C, de Seze J, Zephir H, Paul F, Franciotta D, et al. Frequency and syndrome specificity of antibodies to aquaporin-4 in neurological patients with rheumatic disorders. Mult Scler 2011:17:1067-73

2. Wingerchuk DM, Lennon VA, Pittock SJ, Lucchinetti CF, Weinshenker BG. Revised diagnostic criteria for neuromyelitis optica. Neurology 2006;66:1485-9.

3. Kolefenbach JR, Horner BJ, Ferucci Ed, West SG. Neuromyelitis optica spectrum disorder in patients with connective tissue disease and myelitis. Arthritis Care Res 2011;63:1203-8.

4. Sciascia S, Roccatello D, Rossi D, Rossi A, Mereuta M, Cavallo R. High-titer anti-aquaporin-4-IgG-associated myelitis in rhupus syndrome. J Rheumatol 871-2.

5. Amezcua-Guerra LM. Overlap between systemic lupus erythematosus and rheumatoid arthritis: Is it real or just an illusion? J Rheumatol 2009;36:4-6.

6. Chogle AR, Shah CV, Murthy AK. Role of anti-tumor necrosis factor-alpha blockers in inducing lupus erythematosus tumidus in "rhupus syndrome". J Rheumatol 2011;38:1218-9.

7. Graber DJ, Levy M, Kerr D, Wade WF. Neuromyelitis optica pathogenesis and aquaporin 4. J Neuroinflammation 2008;5:22.

8. Jacob A, Weinshenker BG, Violich I, McLinskey N, Krupp L, Fox $\mathrm{RJ}$, et al. Treatment of neuromyelitis optica with rituximab: retrospective analysis of 25 patients. Arch Neurol 2008;65:1443-8.

9. Mandler RN, Ahmed W, Dencoff JE. Devic's neuromyelitis optica: A prospective study of seven patients treated with prednisone and azathioprine. Neurology 1998;51:1219-20.

J Rheumatol 2012;39:4; doi:10.3899/jrheum.111203 Personal non-commercial use only. The Journal of Rheumatology Copyright @ 2012 . All rights reserved. 